\title{
Transmission des connaissances dans les relations de coopération inter-entreprises : TIC versus face-à-face
}

\section{Knowledge transmission in cooperative relationship between companies : ICT versus face to face}

\author{
ANNE AGUILERA (auteur chargé de la correspondance) \\ Chercheuse \\ Laboratoire Ville, Mobilité, Transport (LVMT) \\ Université Paris Est, INRETS \\ 6-8 avenue Blaise Pascal \\ Champs sur Marne \\ F-77455 Marne la Vallée Cedex 2 \\ aguilera@inrets.fr
}

\section{VIRGINIE LETHIAIS}

Maître de conférences

Télécom Bretagne, M@rsouin (Môle de Recherche sur la Société et les Usages

d'Internet)

Technopôle Brest-Iroise - CS 83818

F-29238 Brest Cedex 3

Mots-clés : TIC, face à face, transmission des connaissances, coopération inter-

entreprises

Keywords : ICT, face to face, knowledge transmission, inter-firms cooperative relationships

Classification JEL: D83, L14, O33, R3. 


\title{
Transmission des connaissances dans les relations de coopération inter-entreprises : TIC versus face-à-face
}

\author{
Knowledge transmission in cooperative relationship \\ between companies : ICT versus face to face
}

\section{Résumé}

L'usage des TIC est régulièrement envisagé comme pouvant favoriser une réduction des besoins de mobilité physique. Cette recherche vise à identifier et hiérarchiser les facteurs qui influencent la fréquence relative des rencontres face à face dans les relations de coopération inter-entreprises. Une analyse économétrique, basée sur une enquête auprès de 348 entreprises bretonnes, nous permet de confirmer l'existence d'une relation positive entre le besoin de partager des informations tacites et les rencontres face à face. Nous montrons aussi que la capacité à codifier les échanges, la distance géographique entre les partenaires, le nombre des relations de coopération et l'appropriation d'Internet dans l'entreprise contribuent à réduire le recours à la mobilité.

Mots-clés : TIC, face à face, coopération inter-entreprises, distance, région Bretagne

\section{Summary}

The use of ICT is regularly regarded as being able to reduce the need for physical mobility. This research aims at identifying and prioritising the factors that influence the relative frequency of face-to-face meetings when companies cooperate with each other. An econometric analysis, based on a survey of 348 companies of the Brittany Region (France) allowed us to confirm that there is a positive relationship between the need to share tacit information and face to face meetings. In addition, we showed that the encoding of exchanges, the geographical distance between the partners, the multiplication of partnerships, and the acceptance of internet by the company all contribute to a reduction in face-to-face meetings.

Keywords: ICT, face to face, inter-firms cooperation, distance, Brittany region 


\section{Introduction}

La nature des facteurs qui expliquent les différences en matière d'adoption et d'usage des Technologies de l'Information et de la Communication (TIC) dans les entreprises est un thème important de la littérature économique. La transmission de connaissances dans les relations inter-entreprises (clients, partenaires, etc.) en constitue un cadre d'analyse spécifique. Les principales questions portent sur l'influence de facteurs comme la localisation (urbaine versus rurale) de l'entreprise (Charlot et Duranton, 2006 ; Galliano et Roux, 2006), le type de partenaire (Amabile et Gadille, 2003), et la nature des connaissances échangées : certaines catégories de connaissances sont considérées comme codifiables, c'est-à-dire transmissibles par les TIC, tandis que d'autres, qualifiées de tacites dans la littérature, ne le sont pas. Leur transmission impliquerait alors par essence la co-présence des interlocuteurs (Ancori et al., 2000 ; Guillain, 2000 ; Johnson et al., 2002).

Ce dernier aspect est toutefois débattu. D'une part, la pertinence mais également l'opérationnalité de la distinction entre connaissance tacite et connaissance codifiée sont régulièrement remises en cause (Balconi et al., 2007). Les discussions portent d'autre part sur l'« automaticité » du lien entre la nature des connaissances à transmettre et le choix d'un vecteur de communication, d'autres facteurs pouvant entrer en ligne de compte dans ce choix. Sont notamment évoqués la connaissance réciproque des interlocuteurs et leur confiance mutuelle (Boschma, 2005 ; Handy, 1995), la nature des technologies de communication effectivement disponibles ainsi que la maîtrise qu'en ont les interlocuteurs. La distance géographique, qui détermine les coûts (monétaires mais aussi psychologiques) d'accès aux interlocuteurs est également invoquée : plus la distance est grande et plus les interlocuteurs seraient amenés à rechercher des solutions de communication à distance et à réduire les interactions en face à face afin de limiter leur mobilité. En revanche, la part du face à face serait plus importante entre des interlocuteurs localisés à proximité, indépendamment de la nature des connaissances (Rallet et Burmeister, 2002). Par ailleurs, le développement de technologies toujours plus proches du face à face (comme la visioconférence, voire certains outils de travail collaboratif), favoriserait pour certains une «spécialisation » des rencontres physiques sur les interactions «les plus complexes » (Charlot et Duranton, 2006 ; Johnson et al., 2004). Enfin, plusieurs phases pouvant généralement être identifiées dans une relation de coopération, et chacune d'entre elles pouvant s'appuyer sur des échanges de connaissances de différente nature, l'arbitrage entre les modes de communication pourrait aussi étroitement dépendre du moment de coopération dans laquelle les partenaires sont impliqués (Kaufmann et al.., 2003, Gallié, 2003).

Finalement, en dépit d'un large consensus sur le fait que la diffusion des TIC dans le monde professionnel ne conduit pas à une disparition pure et simple des rencontres physiques (Gallié et Guichard, 2005 ; Gaspar et Glaeser, 1998; Kalika et al., 2007 ; Morgan, 2004) et donc des déplacements professionnels (Aguiléra, 2008 ; Torre, 2008), 
la question des facteurs qui expliquent, pour une relation donnée, la part des interactions en face à face par rapport aux interactions à distance, nous semble loin d'être tranchée et fait l'objet de cet article. Notre objectif est de déterminer sous quelles conditions l'usage des TIC peut conduire à une limitation du face à face. Pour répondre à cette question, nous avons cherché à identifier et à hiérarchiser les facteurs qui influencent la fréquence relative des rencontres en face à face dans les coopérations inter-entreprises. Ce travail s'efforce par ailleurs de distinguer, au sein des TIC, les technologies traditionnelles (comme le téléphone, le courrier et le fax) des technologies plus avancées (le courriel, la visioconférence, les outils de travail collaboratif ou de gestion des collaborations).

Les données utilisées proviennent de l'enquête « Entreprises et TIC » réalisée en 2006 par le Môle Armoricain de Recherche sur la Société de l'Information et les Usages d'Internet (M@rsouin) auprès de plus de 2000 entreprises bretonnes de 10 à 250 salariés et de plusieurs secteurs d'activité. Un volet de cette enquête interroge spécifiquement les entreprises sur plusieurs aspects de leur relation de coopération la plus importante, une telle relation étant explicitement définie dans le questionnaire comme une relation privilégiée avec un partenaire impliquant un partage d'informations, de connaissances ou de compétences. A l'issue de cette enquête, nous disposons d'un échantillon de 348 entreprises ayant renseigné cette partie du questionnaire.

La première partie de cet article explicite le cadre théorique de cette recherche et ses hypothèses La deuxième partie présente une analyse descriptive des relations de coopération des entreprises bretonnes, mettant notamment en évidence une certaine complémentarité entre les différents outils de communication utilisés. La troisième partie présente les variables et les modèles économétriques. Enfin, la quatrième partie confronte les hypothèses formulées en première partie aux résultats des modèles. Nous validons ainsi l'hypothèse de l'existence d'un lien positif entre la nécessité de partager des informations «tacites » et la fréquence des déplacements professionnels. De plus, nous montrons que la codification des échanges, l'éloignement et la multiplication des partenaires ainsi que l'appropriation d'Internet par l'entreprise vont de pair avec la réduction de la part des rencontres en face à face. La conclusion revient sur ces enseignements et ouvre plusieurs pistes de recherche.

\section{-1 -}

\section{Cadre théorique et hypothèses}

La multi-localisation, l'appartenance à des réseaux d'entreprises nationaux et trans-nationaux, la taille des aires de marché impliquent que beaucoup d'entreprises sont désormais régulièrement confrontées à des besoins de communication (au sens large) avec des interlocuteurs distants et qui sont parfois, avec la mondialisation de l'économie, très éloignés (Amin, 2002 ; Lorentzon, 2003). Les coopérations inter- 
entreprises en constituent une des facettes. Depuis la fin des années soixante-dix, ces relations sont en effet en augmentation sous l'effet des exigences croissantes en matière de flexibilité, de réactivité et d'innovation (Moati, 2002).

Les coopérations inter-entreprises concernent tous les secteurs d'activité et toutes les tailles d'entreprises. Le SESSI (2005) estime par exemple qu'en France, au sein des groupes industriels, plus de huit entreprises productrices sur dix font partie d'un tel système de coopération. Dans le domaine des services aux entreprises, la part des entreprises engagées dans au moins une relation avec une autre entreprise est évaluée à $20 \%$ (Lebon et Ballet, 2004). Ces relations offrent toutefois une grande diversité de forme (par exemple certaines sont contractualisées, d'autres non), de contenu (s'agissant notamment de la fonction sur laquelle porte la coopération: recherche et développement, commercialisation, etc.), de durée ou encore de localisation des partenaires. Cela implique une diversité tout aussi élevée des besoins en termes de coordination et de transmission des connaissances.

\subsection{Face-à-face et nature des connaissances échangées}

Pour analyser les mécanismes de coordinations entre des interlocuteurs, la littérature oppose généralement les TIC (regroupant ainsi en fait une très grande variété d'outils) et le face à face, et accorde une place prépondérante à la nature des connaissances échangées dans les modalités de l'arbitrage entre ces deux «modes » de communication.

La distinction la plus utilisée oppose les connaissances qui sont dites codifiables, c'est-à-dire transmissibles par l'intermédiaire des TIC, et celles qui sont tacites, dont la transmission nécessite au contraire la présence simultanée des interlocuteurs dans un même lieu (Guillain, 2000). Cette seconde catégorie concerne en particulier des connaissances qui doivent être co-construites par les interlocuteurs, des connaissances potentiellement ambiguës, dont la compréhension peut prêter à confusion ou encore des connaissances particulièrement complexes. La classification proposée par B.A. Lundvall et B. Johnson (1994) intègre dans cette catégorie d'une part les compétences et le savoir-faire (know-how), d'autre part les informations sur qui sait quoi et qui sait quoi faire (know-who). Par opposition, les connaissances qui peuvent être codées concernent, toujours selon ces auteurs, d'un côté les connaissances sur les faits (knowwhat), et d'un autre côté les connaissances sur les principes et les lois (know-why).

Cette grille d'analyse suggère est que plus la part des connaissances tacites est importante dans une relation, plus les rencontres physiques sont nécessaires. Sa validation empirique s'avère néanmoins délicate. En effet la distinction entre connaissance tacite et connaissance codifiable est loin d'être évidente et ne fait pas l'unanimité (Balconi, et al., 2007). Les travaux empiriques s'en tiennent d'ailleurs souvent à une typologie peu précise des connaissances opposant deux catégories : les 
informations «complexes » et les informations «basiques », sans forcément beaucoup plus de détail en termes de définition non plus que de mesure. Par exemple, à partir d'une série d'interviews dans l'entreprise Volvo en Suède, S. Lorentzon (2003) confirme que les rencontres en face à face servent à la transmission d'informations qu'il qualifie de non structurées, informelles et sensibles, mais dont il ne précise pas véritablement le contenu. Un autre écueil concerne la mesure précise de la part respective des connaissances tacites et codifiables dans une relation donnée, qui peut varier considérablement selon les phases de cette relation (Vecchi et Wickham, 2008). Il peut donc être délicat de quantifier la part totale des connaissances tacites et codifiables lorsque la relation considérée couvre une période de temps importante.

\subsection{Face-à-face, codification des échanges et appropriation des TIC}

En marge de sa mise en œuvre pratique, cette hypothèse se révèle fragile sur plusieurs autres plans. Ainsi la part de connaissances potentiellement codifiables peut diverger de celle effectivement transmise par les TIC. Le manque d'équipement en TIC dans l'entreprise, mais également une maîtrise insuffisante des outils disponibles constituent des explications. C. Cariou et V. Lethiais (2006) montrent ainsi que la part des connaissances codifiées dans les échanges inter-entreprises est augmentée non seulement par la part de connaissances de type know-what partagée mais aussi de façon conjointe par l'usage qui est fait des TIC. Une autre explication à la divergence entre la part d'information codifiable et celle qui est effectivement transmise à distance peut être, par ailleurs, une non utilisation délibérée (ou une sous-utilisation) des technologies disponibles. On rencontre en particulier ce dernier cas de figure à propos de la visioconférence qui, pour diverses raisons (mauvaise qualité des transmissions, problèmes de fiabilité dans l'équipement, difficulté d'utilisation lorsque les utilisateurs sont nombreux, etc.), est souvent peu utilisée dans les entreprises qui en sont équipées (Aguiléra et al., 2007 ; Arnfalk et Kogg, 2003). Plus largement, l'adoption de certaines technologies au sein de l'entreprise n'implique pas nécessairement leur utilisation, une période d'apprentissage et des changements organisationnels étant souvent un préalable à leur appropriation (Galliano et Roux, 2006, Galliano et al., 2007). Enfin, la nature des outils disponibles n'est pas neutre car tous ne permettent pas la transmission du même type d'information : certaines informations peuvent ainsi s'avérer ou non transmissibles à distance selon le type de technologie à disposition. Il y aurait alors un stade intermédiaire entre informations basiques et informations complexes, les technologies les plus proches du face à face (comme le téléphone et la visioconférence) permettant d'échanger à distance des informations plus sophistiquées que par courrier ou courriel, mais néanmoins moins complexes que celles qui requièrent la co-présence des interlocuteurs. Le partage entre tacite et codifiable dépendrait finalement à la fois des outils TIC disponibles mais aussi de leur usage par les personnes concernées.

Finalement, certaines technologies seraient dans une relation de complémentarité avec le face à face et d'autres dans une relation de substitution. S. Charlot et G. 
Duranton (2006) constatent effectivement une forte complémentarité entre l'usage du téléphone et celui du face à face, tandis que le courriel et le courrier semblent se substituer au face à face (la significativité statistique étant toutefois faible selon les auteurs). Une conséquence serait que la «sophistication» des TIC permettrait une plus grande sélectivité en matière de mobilité, en s'accompagnant d'une spécialisation et donc d'une limitation des interactions face à face aux plus indispensables, c'est-à-dire aux plus complexes (Charlot et Duranton, 2006). La comparaison de l'usage des différents moyens de communication par les entreprises françaises du secteur manufacturier entre 1987 et 1997 dans le cadre de leurs relations externes (clients et fournisseurs) semble confirmer cette hypothèse, puisqu'une légère diminution de l'usage du face à face est mise en évidence, au profit de l'usage de l'ordinateur mais aussi du papier (Charlot et Duranton, 2006). A l'inverse S. Lorentzon (2003) fait dans le cas de Volvo le constat, sur la période 1993-1998, de la stabilité de la part des déplacements professionnels (évalués au total à $1 \%$ des contacts) dans les relations de l'entreprise avec l'extérieur (Lorentzon, 2003). L'hypothèse d'une baisse des déplacements professionnels avec l'usage croissant des TIC est donc loin d'être validée. Le constat est du reste identique pour ce qui concerne les autres motifs de la mobilité (Rallet et al., 2009).

\subsection{Face à face et proximités spatiale et non spatiale des partenaires}

Une autre série de critiques remet plus fondamentalement en cause l'influence dominante de la nature des connaissances sur l'arbitrage entre TIC et face et face, au motif que d'autres facteurs seraient au moins aussi importants dans le choix d'un support de communication. En premier lieu, le lien positif entre la part des connaissances tacites et la part du face à face dans les échanges serait opérant seulement en cas d'éloignement suffisamment important des interlocuteurs, la difficulté de franchissement de la distance impliquant alors une plus grande sélectivité et une plus grande rationalisation de la mobilité (Rallet et Torre, 2004). De fait, M. Le Goff-Pronost et V. Lethiais (2008) montrent de fait, à partir d'une enquête réalisée fin 2003 auprès de 850 PME bretonnes, que les entreprises dont le partenaire est localisé hors de la région Bretagne utilisent plus intensivement les TIC. S. Lorentzon (2003) note pour sa part que la visioconférence est principalement utilisée par les salariés suédois de Volvo lorsqu'ils échangent avec leurs interlocuteurs localisés en Australie, tandis qu'elle est peu mobilisée avec les partenaires suédois mais aussi européens. Ce dernier résultat, s'il va bien dans le sens de l'hypothèse formulée plus haut, pose d'ailleurs aussi la question du seuil à partir duquel les entreprises considèrent qu'elles sont trop éloignées de leur partenaire pour se déplacer... De façon complémentaire, certains auteurs considèrent que la part de la nature des connaissances tacites serait moins opérante dans le cas d'interlocuteurs géographiquement proches, pour lesquels le déplacement est globalement peu coûteux (Rallet et Burmeister, 2002). Par ailleurs, si le coût de la mobilité dépend de la distance entre les interlocuteurs, ce coût est potentiellement 
multiplié par le nombre d'interlocuteurs. On peut ainsi faire une hypothèse supplémentaire qui est que plus le nombre de relations dans lesquelles l'entreprise est engagée est important, plus elle va avoir tendance à faire un plus grand effort de rationalisation du face à face, afin de minimiser le coût global de la mobilité.

Des formes non spatiales de proximité (Boschma, 2005 ; Bouba-Olga et Grossetti, 2008) sont aussi évoquées pour expliquer l'arbitrage entre les modes de communication, en particulier lorsque les interlocuteurs sont éloignés au plan géographique. Les proximités d'essence cognitive, comme la connaissance et la confiance réciproque des interlocuteurs, sont notamment censées rendre plus faciles et aussi plus efficientes les interactions à distance (Gallié et Guichard, 2005 ; Knoben et Oerlemans, 2006). A l'opposé, si les interlocuteurs se connaissent mal, ils vont avoir besoin de plus de se rencontrer, notamment au début de la relation, pour construire la confiance ou encore bâtir des règles communes de fonctionnement (Gallaud et Torre, 2005). En comparant les modes de communication au sein de plusieurs projets d'innovation impliquant des interlocuteurs localisés à distance, J. Hildrum (2007) constate effectivement que la présence de membres ayant la confiance des autres membres pour assurer le rôle de médiateur dans la transmission des connaissances diminue la part relative des rencontres physiques. En pratique toutefois les formes non spatiales de proximité sont difficiles à mesurer et ne font pas complètement consensus. Nous-mêmes aurons recours à deux paramètres qui tentent d'approcher cette notion de proximité non spatiale, et qui sont d'une part l'appartenance au même groupe (au sens juridique), et d'autre part le fait que la relation est contractualisée.

Enfin, le fait de se déplacer peut être considéré comme une marque d'intérêt pour son ou ses interlocuteurs : la part du face à face est donc potentiellement plus élevée dans les relations pour lesquelles existe une asymétrie de statut entre les interlocuteurs (Arnfalk et Kogg, 2003). Cette hypothèse ne pourra cependant pas être testée sur notre échantillon, l'enquête ne renseignant pas le volume des déplacements qui relèvent de l'entreprise enquêtée et celui qui relève de son partenaire.

Au terme de cette revue de littérature, deux questions émergent et font l'objet de l'analyse empirique. La première concerne la nature des facteurs qui expliquent la part de rencontres en face à face dans les relations de coopérations inter-entreprises. Les facteurs que nous allons considérer sont la nature des connaissances échangées, la capacité de codification des échanges (y compris l'appropriation des TIC au sein de l'entreprise), plusieurs mesures de la proximité entre les partenaires, et enfin le nombre de partenaires avec lesquels l'entreprise entretient une coopération.

La seconde question porte sur l'impact de la distance géographique sur ces mêmes déterminants. Notre hypothèse est que la part du face à face dépend de la nature des connaissances échangées seulement lorsque les interlocuteurs sont suffisamment éloignés spatialement. 


\section{Les relations de coopération des entreprises bretonnes : une analyse descriptive}

\subsection{Les données}

En l'absence de données nationales permettant de tester ces hypothèses, nous avons utilisé une enquête à laquelle nous avons participée dans le cadre du réseau M@rsouin. Cette enquête a été réalisée en 2006 auprès de 2000 entreprises bretonnes de 10 à 250 salariés des secteurs de l'industrie (hors industrie agro-alimentaire), du commerce et des services. Elle comporte un volet qui décrit les coopérations avec d'autres entreprises et qui permet notamment de mesurer la fréquence du face à face et la fréquence d'usage de plusieurs outils de communication à distance.

Le questionnaire définit une relation de coopération comme une relation privilégiée avec un partenaire impliquant un partage d'information ou de compétences. Cela correspond à des relations de type réponse commune à un appel d'offre, relation de sous-traitance, développement d'un produit commun, mise en place d'une structure commune, etc. Les relations de type prestations de services standards ou fournitures de biens sur catalogue sont explicitement exclues.

Chaque entreprise a été interrogée sur sa principale relation de coopération, cette dernière étant définie comme la plus importante en termes de partage d'information. Ce volet du questionnaire nous permet d'identifier, pour les 348 entreprises ayant déclaré une relation de coopération, différentes caractéristiques du partenaire, la nature des informations échangées, les outils de communication utilisés ainsi que leur fréquence d'utilisation, et enfin un certain nombre des difficultés éventuelles rencontrées dans le cadre de cette relation en matière de transmission des connaissances. La proximité spatiale ainsi que certaines formes de proximité non spatiales avec le partenaire peuvent également être identifiées.

Nous montrons d'abord que dans la majorité des cas le partenaire principal est situé hors de la région Bretagne, et aussi que ce résultat peut être expliqué par le fait que ce sont bien plus des proximités non spatiales qui comptent dans le choix d'un partenaire, que la stricte proximité géographique. Ce résultat fait d'ailleurs écho à d'autres travaux récents (Ferru, 2010). Ensuite, nous évaluons la part de chacune des catégories de connaissances échangées sur la base de la typologie proposée par B.A. Lundvall et B. Johnson (1994). Enfin, nous analysons à la fois l'usage des différents moyens de communication répertoriés dans le questionnaire, ainsi qu'un certain nombre de leurs relations.

\subsection{Formes de proximité entre les partenaires}


Si elle ne fournit pas d'indication sur la distance kilométrique ou temporelle entre les interlocuteurs, l'enquête nous donne néanmoins une bonne mesure de la proximité spatiale entre l'entreprise et son partenaire selon une échelle qui compte cinq niveaux. Le partenaire peut ainsi être localisé dans la même zone d'activité, à l'échelon local, à l'échelon régional, à l'échelon national et enfin à l'échelon international.

Les résultats de l'analyse de la localisation des partenaires des entreprises bretonnes ne démentent pas ceux d'autres études qui ont mis en évidence le faible besoin de proximité géographique dans les relations inter-entreprises (Bathelt et al., 2004). Pour plus de la majorité (61\%) des 348 entreprises interrogées, la relation de coopération principale implique en effet un partenaire situé hors de la région. L'échelon national reste néanmoins prépondérant pour les entreprises bretonnes : dans $43 \%$ des cas le partenaire est situé hors de la Bretagne mais en France, et dans seulement 18\% il est localisé à l'étranger. La situation excentrée de la région n'est probablement pas étrangère à ce résultat.

La prééminence des coopérations extérieures à la région n'est par ailleurs pas démentie lorsque l'on prend en compte non plus la relation de coopération principale mais l'ensemble des relations de coopérations : en effet $66 \%$ des entreprises bretonnes déclarent avoir plus de la moitié de leurs partenaires hors de la Bretagne. Plus précisément, lorsque le partenaire principal est hors de la région, en général les autres partenaires le sont aussi.

Les résultats de l'enquête confirment par ailleurs que le choix d'un partenaire est bien plus lié à des critères de proximité organisationnelle (au sens du partage de règles organisationnelles communes) et cognitive qu'à une proximité d'ordre seulement géographique (Ferru, 2010 ; Filippi et Torre, 2000 ; Rallet et Torre, 2004) : en effet plus de la moitié des entreprises bretonnes évoquent l'appartenance au même réseau relationnel comme facteur de choix, alors que seulement le quart d'entre elles mentionne la proximité spatiale.

L'exploitation d'une autre question, qui évoque plusieurs sources de difficultés possibles dans la relation avec le partenaire, permet de mieux comprendre ces résultats. On constate en effet que le fait d'être éloigné au plan géographique est rarement considéré comme une difficulté dans les relations inter-entreprises : seule une minorité des entreprises enquêtées (22\%) déclare que l'éloignement constitue un frein dans les échanges avec le partenaire. En revanche, elles sont sensiblement plus nombreuses à mentionner l'absence de certaines formes de proximité non spatiale comme problématique. Le manque de compatibilité entre les équipements technologiques, l'absence de culture, de code ou de langage commun et la divergence dans le mode d'organisation ressortent comme des difficultés dans les échanges pour respectivement $27 \%, 22 \%$ et $31 \%$ des entreprises. 


\subsection{Nature des connaissances}

Le questionnaire différencie quatre «types » de connaissances qui font référence à la typologie de B.A. Lundvall et B. Johnson (1994) : des informations liées à l'activité des entreprises, du savoir scientifique fondamental, du savoir faire pratique et enfin des compétences ou expertises particulières. Comme l'indique le tableau 1, les informations potentiellement codifiables sont la composante majeure des échanges dans les relations de coopération des entreprises bretonnes. Parmi elles, les connaissances de type knowwhat (informations liées à l'activité) sont de surcroît largement prépondérantes par rapport aux connaissances de type know-why (savoir scientifique fondamental). Le partage de connaissances tacites est néanmoins important, environ la moitié des entreprises déclarant échanger beaucoup de connaissances de type know-how (savoir faire pratique et compétences ou expertises particulières).

(Tableau 1)

\subsection{Usage et complémentarité des outils de communication}

L'enquête propose deux mesures complémentaires de l'usage des différents moyens de communication. La première s'appuie sur le ressenti des entreprises, la question portant sur l'importance des rencontres physiques, des technologies traditionnelles (téléphone, minitel, courriel, fax) et enfin des «nouvelles » TIC (courriel, visioconférence, outils de travail collaboratifs, etc.), que nous qualifierons par la suite aussi de technologies avancées ou récentes. La seconde mesure, plus objective, renseigne sur la fréquence d'utilisation des différents moyens ou outils de communication que sont le face-à-face, téléphone, le courrier ou le fax, la messagerie électronique, la visioconférence, les outils de travail collaboratifs (base de données partagées, partage de fichiers, forums, etc.) et enfin les outils de gestion de gestion des collaborations (agendas partagés, listes de diffusion, outils de gestion des tâches, etc.).

$\mathrm{Au}$ classement des modes de communication selon leur importance (outil principal, secondaire ou le moins important), les technologies récentes et les rencontres physiques font quasiment jeu égal : elles sont considérées comme un outil principal de communication avec le partenaire pour près de la moitié des entreprises (voir tableau 2). Ce score important se situe toutefois en deçà de celui des technologies traditionnelles, mentionnées comme l'outil principal de communication par plus des deux tiers de l'échantillon. Ces technologies, de par leur large diffusion et leur facilité d'utilisation, demeurent donc encore largement dominantes dans les échanges entre les entreprises.

(Tableau 2)

Si le face à face est moins souvent l'outil principal de communication que les TIC traditionnelles, il est en revanche plus fréquemment mentionné en tant qu'outil secondaire, ce qui n'est pas le cas pour les TIC récentes : pour 22\% des entreprises 
interrogées ces dernières sont en effet le vecteur de communication le moins important, contre seulement $11 \%$ pour le face à face. Les TIC traditionnelles et le face à face sont ainsi quasiment aussi souvent l'outil principal ou secondaire de communication dans les relations de coopération inter-entreprises.

Le fait que les technologies avancées sont plus fréquemment considérées comme l'outil le moins important dans les communications inter-entreprises est à rapprocher d'une assez faible familiarité des entreprises interrogées avec les outils récents de communication et d'information : en effet un autre volet de l'enquête montre qu'en moyenne seulement $40 \%$ des salariés des entreprises concernées utilisent au moins une fois par semaine le courrier électronique, et 39\% utilisent au moins une fois par semaine Internet. Un quart des entreprises est de fait connecté depuis moins de trois ans à Internet et seulement $40 \%$ le sont depuis plus de cinq ans.

L'analyse en termes de fréquence d'usage des différents outils valide et permet de préciser les résultats précédents sur plusieurs points.

(Tableau 3)

Le tableau 3 indique en effet que les rencontres physiques mais aussi les TIC traditionnelles (téléphone, courrier, fax) ne sont que très rarement jamais utilisées pour communiquer avec le partenaire. Parmi les technologies récentes, seul le mail est dans le même cas de figure, avec un taux de non utilisation de l'ordre de seulement $11 \%$, alors que ce taux oscille entre $45 \%$ (pour les outils collaboratifs) et $87 \%$ (pour la visioconférence) pour les trois autres outils proposés. Ces scores reflètent une très faible pénétration de ces technologies, et en particulier de la visioconférence, dans les entreprises bretonnes, plus qu'une non utilisation qui serait spécifique au contexte des coopérations inter-entreprises.

Le téléphone, le courrier et également le mail sont le plus souvent des outils de communication de fréquence au moins hebdomadaire, et même souvent quotidienne : entre $40 \%$ et $51 \%$ des entreprises interrogées déclarent les utiliser tous les jours pour communiquer avec leur partenaire. Le franchissement de la distance reste par contre un obstacle au face à face qui fait à l'évidence l'objet de rationalisations qui ne concernent pas, ou en tout cas à une échelle de temps différente, les communications à distance, aisées et peu coûteuses : ainsi les rencontres physiques sont, dans les deux tiers des cas, au mieux seulement mensuelles. On verra que le fait que la majorité des partenaires est situé hors de la région contribue à expliquer ce chiffre.

Des tests de Khi-Deux permettent de préciser la nature des reklations entre les différents modes de communication, et de faire apparaître une forte complémentarité entre les technologies traditionnelles et le face à face. C'est conforme aux résultats obtenus par S. Charlot et G. Duranton (2006) pour les entreprises françaises du secteur de la manufacture, et, plus largement, aux travaux menés dans le domaine de 
l'économie des transports depuis plusieurs décennies concernant les relations entre les TIC et la mobilité (pour motif personnel) des individus (Claisse et Rowe, 1993 ; Ling et Haddon, 2001 ; Ling et Yttri, 2002 ; Massot, 1995).

Nous constatons par ailleurs un effet de substitution entre les technologies avancées et les technologies traditionnelles, ce qui est là encore conforme aux résultats obtenus par S. Charlot et G. Duranton (2006). En revanche, la relation entre l'importance des technologies récentes et le face à face n'apparaît pas clairement.

La même analyse, appliquée cette fois aux fréquences d'utilisation des différents outils, confirme la relation de complémentarité mentionnée plus haut entre TIC traditionnelles et rencontres physiques. En effet, plus l'entreprise déclare utiliser fréquemment le téléphone et le courrier, plus elle a recours au face à face. En revanche, le mail semble se substituer au face à face, son usage très fréquent étant associé à des rencontres physiques moins fréquentes. Cet outil permettrait donc, dans certaines relations de coopération, de réduire la mobilité, conformément là encore aux résultats obtenus dans un tout autre contexte par S. Charlot et G. Duranton (2006). La visioconférence apparait en revanche comme étant complémentaire aux rencontres physiques. Ce résultat s'explique par le très faible nombre d'entreprises qui utilisent cette technologie, qui sont caractérisées par un besoin important de relations de face à face.

Plusieurs modèles économétriques vont à présent nous permettre de préciser ces premiers résultats, en introduisant notamment comme paramètre explicatif la distance géographique entre les partenaires, la nature des échanges et le niveau d'appropriation des TIC dans l'entreprise enquêtée.

\section{Présentation des modèles}

Afin de tester les raisons du recours à la mobilité physique dans les relations de coopération, nous avons construit trois modèles économétriques, dont les résultats sont présentés dans la quatrième partie. Nous décrivons ici la variable expliquée et les variables explicatives, dont le choix fait directement écho aux hypothèses formulées en première partie, ainsi que les modèles économétriques.

\subsection{La variable expliquée et les modèles}

\section{La variable expliquée}

Comme nous l'avons indiqué dans la partie précédente, nous disposons dans l'enquête de deux mesures du recours aux rencontres physiques dans la principale relation de coopération des entreprises interrogées : la première est l'importance du face 
à face (en comparaison aux technologies traditionnelles et récentes), et la seconde exprime une fréquence de déplacements. Nous avons choisi d'utiliser cette dernière car elle est plus objective.

La fréquence d'utilisation de chaque moyen de communication a été codée de la manière suivante : 4 si l'entreprise utilise l'outil quotidiennement, 3 si elle l'utilise de façon hebdomadaire, 2 si elle en a un usage mensuel, 1 si elle l'utilise moins de une fois par mois et enfin 0 si elle n'utilise jamais cet outil.

Notre objectif étant de déterminer dans quels cas de figure d'autres modes de communications se substituent à la mobilité physique, nous avons choisi de nous intéresser à la fréquence du face à face dans les échanges avec le partenaire principal, relativement à tous les autres modes de communication. Nous avons ainsi calculé pour chaque entreprise la fréquence relative des rencontres physiques qui est égale à la fréquence des rencontres physiques rapportée à la fréquence d'utilisation moyenne de l'ensemble des outils de communication.

$$
x=\frac{f_{\text {Re ncontres }}}{\frac{1}{7} \sum_{i=1}^{i=7} f_{i}},
$$

où $\mathrm{i}=1$ à 7 correspond aux outils suivants: les rencontres physiques, le téléphone, le courrier ou fax, la messagerie électronique, la visioconférence, les outils de travail collaboratif et les outils de gestion des collaboration.

A partir de $\mathrm{x}$ nous avons ensuite construit une variable ordonnée en 8 modalités qui prend les valeurs suivantes :

$$
\left\{\begin{array}{lll}
y=1 & \text { si } & x \leq 0.25 \\
y=2 & \text { si } & 0.25<x \leq 0.5 \\
y=3 & \text { si } & 0.5<x \leq 0.75 \\
y=4 & \text { si } & 0.75<x \leq 1 \\
y=5 & \text { si } & 1<x \leq 1.25 \\
y=6 & \text { si } & 1.25<x \leq 1.5 \\
y=7 & \text { si } & 1.5<x \leq 1.75 \\
y=8 & \text { si } & x>1.75
\end{array}\right.
$$

La distribution de la variable y est présentée dans le graphique 1.

\section{(Graphique 1)}

Cette variable va être estimée dans la quatrième partie à l'aide d'un modèle Logit ordonné. 


\section{Les modèles Logit ordonnés}

La variable y, qui mesure la fréquence relative des rencontres en face-à-face regroupée en 8 modalités, a été estimée à l'aide d'un modèle Logit ordonné, sur trois échantillons différents.

Le premier modèle estime la fréquence relative du face à face pour l'ensemble des entreprises de notre échantillon ayant identifié une relation de coopération. Il s'agit de déterminer, parmi les facteurs identifiés dans la revue de la littérature, lesquels ont effectivement une influence sur les besoins de mobilité physique.

Les modèles 2 et 3 cherchent à vérifier si la distance spatiale entre les interlocuteurs ne remet pas partiellement en cause l'influence des précédents facteurs. Dans le modèle 2, nous n'intégrons ainsi que les entreprises ayant une relation de coopération de proximité (au sein de la même région) et dans le modèle 3 seulement celles ayant une relation distante (avec un partenaire localisé en dehors de la région), et nous testons pour chacun de ces deux modèles la significativité des variables explicatives introduites dans le premier modèle.

\subsection{Les variables explicatives}

Conformément aux hypothèses formulées en première partie, les variables explicatives sont les suivantes : la nature des connaissances échangées, le niveau de codification des échanges, les formes de proximité (notamment spatiale) avec le partenaire, l'utilisation d'autres modes de communication, et enfin l'appropriation des TIC par l'entreprise enquêtée.

\section{Nature des connaissances et vecteurs des échanges}

Afin de tester les hypothèses formulées dans la première partie concernant le rôle de la nature des connaissances échangées et la possibilité effective de les codifier, nous avons construit plusieurs variables. Nous n'avons pas ici fait d'hypothèse sur le lien entre les différents types de connaissances et la possibilité ou non de les codifier. Nous avons au contraire choisi de mesurer l'impact d'une part de la nature des connaissances et d'autre part de leur niveau de codification sur la part relative du face à face.

L'enquête indique, pour chaque entreprise, l'importance de chacune des catégories de connaissances échangées (informations liées à l'activité, savoir scientifique fondamental, savoir faire pratique, compétences ou expertises particulières), que nous avons codé 3 pour beaucoup, 1 pour un peu et 0 pour pas du tout (voir tableau 1). Nous avons alors ramené chacune de ces valeurs à la somme de ces valeurs afin de disposer, pour chaque catégorie, d'une variable qui approxime la part de cette catégorie dans l'ensemble des échanges avec le partenaire. 
Par ailleurs, les entreprises ont été interrogées sur l'utilisation de deux différents vecteurs d'échanges que sont les documents et fichiers d'une part et les échanges oraux d'autre part. Deux variables ont alors été introduites, qui mesurent l'importance de chacun de ces vecteurs : elles prennent la valeur 1 si ce vecteur est beaucoup utilisé et la valeur 0 dans le cas contraire. Elles rendent compte du niveau de codification des échanges entre les partenaires.

\section{Proximités avec le partenaire}

La distance géographique à parcourir ayant probablement une influence sur la décision de se déplacer, une variable de localisation géographique du partenaire a été intégrée dans le modèle, selon les cinq niveaux suivants, directement issus des réponses au questionnaire: même zone d'activité, échelon local, échelon régional, échelon national et échelon international.

L'enquête nous permet par ailleurs de mesurer des formes non spatiales de proximité entre les partenaires, qui sont supposées favoriser les interactions à distance. Nous avons ainsi considéré le fait d'appartenir au même groupe (au sens juridique) comme une telle forme de proximité, et intégré dans le modèle une variable qui prend la valeur 1 si le partenaire appartient au même groupe et 0 sinon. N'ayant en revanche pas de variable permettant directement de mesurer la confiance entre les interlocuteurs, nous avons considéré le fait que la relation soit contractualisée comme un substitut à cette confiance. La variable «contractualisation», qui prend la valeur 1 si la relation est contractualisée et 0 sinon, a donc été introduite dans le modèle afin de déterminer si le fait de fixer des règles qui explicitent les droits et devoirs des deux parties contribue à expliquer l'usage relatif des différents modes de communication.

\section{Nombre de relations de coopération}

$\mathrm{Au}$ même titre que l'éloignement géographique entre les partenaires, qui augmente le coût des rencontres physiques, la multiplication des relations partenariales peut inciter les entreprises à n'avoir recours au déplacement que lorsqu'il est vraiment nécessaire. Par ailleurs, la multiplication des relations partenariales peut pousser l'entreprise à mettre en place des outils permettant de faciliter les relations à distance. Nous avons alors introduit dans le modèle une variable qui prend la valeur 1 si l'entreprise a identifié plus de 10 relations de coopération (ce qui correspond à la valeur médiane du nombre de relations de coopérations) et à 0 sinon.

\section{Importance des autres outils de communication}

Afin de valider et de compléter les résultats obtenus dans l'analyse descriptive sur la complémentarité entre les différents outils et modes de communication, nous avons intégré deux variables dans le modèle. Les variables «importance des technologies traditionnelles » et «importance des nouvelles technologies» prennent chacune la 
valeur 1 si l'entreprise a cité ce mode comme étant l'outil principal pour échanger avec son partenaire, et la valeur 0 sinon.

\section{Appropriation des TIC au sein de l'entreprise enquêtée}

L'utilisation des technologies récentes et donc la possibilité pour les entreprises de substituer certains outils aux rencontres physiques, étant conditionnée à leur appropriation au sein de l'entreprise, nous avons intégré dans le modèle deux variables qui caractérisent cette appropriation. L'appropriation nécessite, dans un premier temps, une période d'apprentissage aux différents outils, et en particulier à Internet, qui conditionne l'utilisation d'une grande partie des technologies que nous avons qualifiées de nouvelles. La variable «Apprentissage d'Internet» prend ainsi la valeur 1 si l'entreprise est connectée depuis plus de 3 ans, et la valeur 0 sinon.

L'appropriation de ces technologies nécessite de surcroît qu'elles soient largement accessibles au sein de l'entreprise et par conséquent que les employés soient familiarisés avec l'outil informatique. Nous avons pour cette raison introduit une variable « diffusion de l'ordinateur » qui mesure la part des salariés au sein de l'entreprise qui utilise au moins une fois par semaine un ordinateur.

\section{Les déterminants du besoin de face à face dans les coopérations inter- entreprises : résultats}

Le tableau 4 présente, pour chacun des trois modèles, les coefficients des différentes variables et les étoiles indiquent le degré de significativité des variables (une étoile pour $10 \%$, deux pour $5 \%$ et trois pour $1 \%$ ). Un coefficient négatif (respectivement positif) implique que cette variable (ou le fait d'appartenir à cette modalité, par rapport à la modalité de référence, notée «Réf») joue négativement (respectivement positivement) sur la fréquence relative des rencontres physiques dans les échanges interentreprises.

(Tableau 4)

\subsection{Le cas général}

L'objectif du premier modèle, qui prend en compte 339 entreprises bretonnes ${ }^{1}$, est de valider notre première hypothèse, c'est-à-dire de vérifier la pertinence de chacune des variables explicatives décrites dans la partie précédente. Au préalable, un certain

\footnotetext{
${ }^{1}$ Certaines entreprises n'ayant pas répondu à la totalité des questions, nous n'avons pu exploiter dans les 3 modèles que les réponses de 339 entreprises parmi les 348 ayant identifié une relation de coopération.
} 
nombre d'autres paramètres, comme le secteur d'activité et la taille de l'entreprise, ont été testés et se sont révélés, conformément à nos attentes, non significatifs sur le plan statistique. Ils n'ont donc pas été introduits dans les différents modèles.

Dans le cas général (modèle 1), les principales variables significatives sont les vecteurs des échanges, la nature des connaissances échangées, la distance spatiale entre les partenaires et enfin les variables d'appropriation des technologies. Les signes des coefficients sont en outre conformes à nos attentes.

La proximité géographique du partenaire augmente la part du face à face. Il est de plus intéressant de noter que cette notion de proximité se définit ici comme une localisation dans la même zone d'activité ou la même localité. En effet, on observe qu'au-delà de ce seuil, le fait que le partenaire soit dans la même région ou ailleurs en France n'a pas d'influence sur la fréquence relative des déplacements (par rapport à une localisation du partenaire à l'échelle internationale). En termes de temps de transport, cette référence à l'échelon local peut être interprétée comme un trajet qui ne dépasse pas la demi-journée (aller-retour), seuil temporel déjà mis en évidence dans d'autres travaux sur les relations inter-entreprises (Arabeyre-Petiot, 1999). Au-delà, les coûts de franchissement de la distance semblent suffisamment importants pour être de nature à limiter les déplacements. L'éloignement incite donc à une rationalisation de la mobilité et conduit corrélativement à un accroissement de la part des communications à distance.

Le nombre de relations de coopération dans lesquelles l'entreprise est impliquée joue lui aussi à la baisse sur le poids relatif des rencontres physiques. De fait la localisation des partenaires est fortement corrélée à celle du partenaire principal : autrement dit si ce dernier est éloigné, la plupart des autres le sont généralement aussi. Le fait d'avoir de nombreux partenaires, pour la plupart éloignés, incite au final les partenaires à réduire la part des déplacements dans les échanges avec le partenaire.

Il semble cependant que les entreprises, dans ce processus de rationalisation, substituent plus les nouvelles technologies que les technologies traditionnelles aux rencontres face à face. Le fait de considérer les nouvelles technologies comme un outil principal dans la relation exerce en effet un impact négatif sur la fréquence de rencontres physiques. Certaines technologies avancées se substituent donc au face à face dans les relations de coopération. En revanche, la variable qualifiant l'importance des technologies traditionnelles n'est pas significative dans ce premier modèle, ce qui ne nous permet pas de confirmer l'effet de superposition, voire dans certains cas d'induction, des technologies traditionnelles sur la mobilité mis en évidence dans d'autres travaux (Kalika et al., 2007).

Le modèle 1 confirme par ailleurs le lien positif entre la part des informations tacites dans les échanges et la nécessité des rencontres en face à face. Une augmentation de la part des informations ou du savoir scientifique dans les échanges conduit en effet à 
diminuer la part des aux rencontres physiques. Si l'on reprend ici la typologie de B.A. Lundvall et B. Johnson (1994), ce résultat valide le fait que l'échange de connaissances de types know-what ou know-why peut plus facilement s'appuyer sur d'autres modes de communications que les rencontres physiques, contrairement aux connaissances de type know-how qui nécessitent le face à face. Toutefois, nos résultats suggèrent qu'une augmentation de la part de savoir faire pratique dans les échanges relativement aux compétences/expertises particulières conduit elle aussi à une diminution de la fréquence du face-à-face. Ces deux catégories de connaissances, qui relèvent du know-how, ne semblent donc pas être identiques en termes de support de communication. La distinction entre le savoir faire pratique et les compétences/expertises particulières étant cependant difficile à saisir, ce résultat demeure complexe à interpréter.

Les liens entre la part de la mobilité et les aspects d'ordres technique mais aussi cognitif relevant des possibilités de codification des connaissances sont en revanche plus explicites. Le modèle 1 montre en effet que les possibilités de codification des échanges sont un facteur limitant des déplacements : l'importance des documents et fichiers dans la transmission des connaissances réduit la part des rencontres physiques. Bien que plusieurs technologies (traditionnelles ou avancées) permettent de transporter la voix, les échanges oraux s'appuient encore de manière significative sur le face à face. Il semble donc que la rationalisation des déplacements et le recours à d'autres modes d'échanges soit conditionnée par la possibilité de substituer aux échanges oraux d'autres vecteurs d'échanges, qui eux nécessitent un effort de codification des connaissances.

Enfin, les variables relatives au niveau de pénétration de l'informatique et d'Internet dans l'entreprise (la maîtrise et l'utilisation des outils informatiques et d'Internet étant un préalable à la faisabilité de l'échange de fichiers) sont significatives sur un plan statistique et ont bien de surcroît le signe attendu. La fréquence relative de la mobilité est en effet diminuée par le fait que l'entreprise est connectée à l'Internet depuis au moins trois ans. Le fait que plus de la moitié des salariés utilisent un ordinateur au moins une fois par semaine joue également à la baisse sur la part relative du face à face. Autrement dit, les capacités cognitives de codification des connaissances sont favorables à la limitation des rencontres physiques, et augmentent corrélativement l'usage des TIC.

Le dernier enseignement du modèle général est la non significativité de deux variables : la contractualisation de la relation et le fait que le partenaire appartienne au même groupe, qui rendent compte d'un certain degré de proximité non spatiale entre les acteurs. Ce constat ne permet cependant pas d'invalider l'hypothèse selon laquelle les proximités non spatiales comptent dans l'arbitrage entre le face à face et les autres modes de communication, car les paramètres mesurant ces proximités restent grossiers, surtout s'agissant de la référence à la contractualisation, qui recouvre une grande diversité de formes. Plus intéressant nous semble le fait que l'appartenance au même 
groupe n'ait ici pas d'effet alors que d'autres travaux ont montré que le recours à la mobilité était moins fréquent dans ce cas de figure (Lorentzon, 2003).

L'enseignement principal de ce premier modèle est qu'il confirme l'hypothèse selon laquelle il faut relativiser le rôle de la nature des connaissances dans l'arbitrage entre TIC et mobilité. Les résultats permettent en particulier de mettre en évidence le rôle d'une part de la possibilité de codification des connaissances, notamment via le niveau d'apprentissage des TIC dans l'entreprise, et d'autre part celui de la distance géographique entre les partenaires. C'est ce dernier point que les deux autres modèles vont à présent nous permettre de mieux expliciter.

\subsection{L'influence de la distance spatiale entre les partenaires}

Les modèles 2 et 3 ont pour objectif d'affiner l'analyse des déterminants de l'usage relatif du face à face dans les relations de coopérations, en érigeant le coût de franchissement de l'espace au statut de paramètre fondamental du choix entre communication à distance et communication en face à face (hypothèse 2). Deux modèles ont ainsi été construits, qui reprennent chacun strictement les mêmes variables que celles du modèle 1 (dont il s'agit d' « affiner » les conclusions), mais pour deux sous populations d'entreprises définies en fonction de la distance au partenaire principal. Dans le modèle 2 les partenaires appartiennent à la même région, tandis que le modèle 3 ne prend en compte que les cas où le partenaire est situé en dehors de la région (ailleurs en France ou à l'étranger).

La lecture du tableau 4 montre que si le nombre de variables statistiquement significatives est réduit par rapport au modèle général, ces deux modèles sont néanmoins riches d'enseignements qui viennent corroborer notre seconde hypothèse et préciser les conclusions du modèle général.

Le premier enseignement est que l'éloignement entre les partenaires n'influence la fréquence relative du face à face que lorsque les relations de coopération s'inscrivent à l'intérieur de l'espace régional. En effet, dans le modèle 3, la variable qui mesure la distance entre les entreprises n'est pas significative, alors que dans le modèle 2, une localisation dans la même zone d'activité se traduit par une fréquence accrue des rencontres physiques. L'éloignement n'a donc plus d'effet au-delà du seuil régional, et le face-à-face ne sera favorisé que par une très forte proximité géographique dans le cas des relations régionales. En revanche, pour les relations extra-régionales, la multiplication des partenariats compte et incite à diminuer la part du face à face dans les échanges avec le partenaire principal.

L'enseignement principal est toutefois que les modèles 2 et 3 aboutissent à des résultats différents concernant le rôle de la nature des connaissances échangées. Le modèle 3 met en évidence les mêmes résultats que le modèle 1, qui nous ont permis de 
valider l'hypothèse de rationalisation des déplacements lorsque les échanges sont principalement basés sur des connaissances codifiables (de type know-what et knowwhy). Le modèle 2 suggère, en revanche, que seule une part importante de connaissances de types know-what (informations liées à l'activité de l'entreprise) se traduit par une réduction de la fréquence des déplacements lorsque la coopération opère à l'échelle infra-régionale. Autrement dit, la rationalisation du face à face concerne plusieurs types de connaissances lorsque le partenaire est distant, alors qu'elle se fait uniquement sur les informations les plus facilement codifiables lorsque le partenaire est proche. L'hypothèse 2 est ainsi validée : lorsque les partenaires sont proches (dans la même région), d'autres modes de communications tendent à se substituer au face à face uniquement lorsque les connaissances échangées sont très facilement codifiables. A l'inverse, lorsque le partenaire est éloigné (en dehors de la région), la rationalisation des déplacements concerne aussi des types de connaissances plus complexes, nécessitant l'utilisation d'outils de communications plus élaborés.

C'est d'ailleurs cohérent avec le fait que dans le modèle 3 la variable mesurant l'utilisation des nouvelles technologies est significative et a un coefficient négatif, ce qui n'est pas le cas dans le modèle 2. Les entreprises dont le partenaire est situé en dehors de la région ont donc tendance à substituer ces technologies aux rencontres en face à face, alors qu'on n'observe pas cette tendance dans le cas des relations infrarégionales.

Enfin, il est intéressant de noter que les deux variables qui permettent de mesurer le niveau d'appropriation des technologies sont significatives dans le modèle 2 et ne le sont pas dans le modèle 3 . Il semble donc que la réduction des relations de face à face soit conditionnée à l'appropriation des technologies au sein de l'entreprise, uniquement dans le cas de relations régionales. Ce résultat s'explique par le fait que les entreprises impliquées dans des relations de coopérations suprarégionales sont généralement familiarisées avec les outils informatiques et avec Internet, qui ne représentent donc pas un facteur «bloquant» dans l'utilisation des nouvelles technologies comme substitut aux relations de face à face.

\section{Conclusion}

L'intensification de l'usage des TIC et la sophistication des outils disponibles sont régulièrement envisagés comme pouvant favoriser, à court ou moyen terme, une réduction des besoins de mobilité physique. Dans le monde professionnel, la traduction de cette hypothèse conduit à envisager qu'une partie des communications professionnelles traditionnellement effectuées en face à face, et spécifiquement celles dont le contenu est le moins complexe, peut être remplacée sans dommage par des échanges à distance. La part des rencontres physiques dans les relations professionnelles diminuerait ainsi avec la part des échanges pouvant être effectivement codifiés. 
Cet article a examiné cette hypothèse dans le cadre spécifique des coopérations inter-entreprises, à partir d'une base de données provenant d'une enquête réalisée en 2006 auprès d'un échantillon représentatif d'entreprises bretonnes. Pour cela nous avons cherché à expliquer la fréquence relative des rencontres physiques dans les relations de coopération en fonction de paramètres identifiés à l'issue d'une revue de la littérature. Cette dernière nous a amené à considérer, outre la nature des connaissances échangées, la distance entre les interlocuteurs ou encore les capacités techniques et cognitives d'utilisation des différentes technologies de communication à distance.

Nous avons alors construit trois modèles économétriques. Le premier, qui prenait en compte l'ensemble des entreprises ayant déclaré une relation de coopération, nous a permis de montrer la pertinence des paramètres retenus. Conformément à l'hypothèse formulée dans la revue de la littérature, la nature des connaissances n'est pas l'unique facteur qui détermine le choix du mode de communication entre les entreprises. La distance et la capacité de codification des connaissances et d'appropriation des technologies apparaissent aussi comme des facteurs déterminants dans la part des interactions en face-à-face dans les relations inter-entreprises. Les deux autres modèles, construits sur deux sous populations d'entreprises de notre échantillon, nous ont permis de préciser l'influence de la distance dans l'arbitrage TIC/mobilité. Les résultats montrent en effet que l'éloignement des partenaires limite les rencontres physiques aux interactions les plus complexes, tandis que lorsque les interlocuteurs sont proches (dans la même région), la nature des connaissances influence moins la fréquence relative du face à face. Ces modèles montrent par ailleurs que, si le fait de considérer les nouvelles technologies comme l'outil de communication principal avec son partenaire implique une diminution du face-à-face dans le cas des relations «distantes », cela n'a pas d'impact lorsque l'on s'intéresse à des relations régionales. La diffusion des outils informatiques et l'apprentissage à Internet au sein de l'entreprise apparaissent en revanche comme des facteurs déterminants de la rationalisation du face-à-face dans les relations au sein de la même région.

En résumé, c'est plus l'appropriation des outils et la capacité à codifier les connaissances échangées que la nature des connaissances échangées qui vont conduire les entreprises à rationaliser leurs déplacements professionnels en leur substituant d'autres modes de communication, dans le cas de relations au sein de la même région. A l'inverse, les entreprises ayant des relations avec des partenaires situés en dehors de la région semblent mieux maîtriser les outils qui leur permettent de communiquer à distance et donc de rationaliser la mobilité et réservent leurs rencontres en face-à-face à des échanges qui rendent le déplacement nécessaire, notamment ceux qui s'appuient sur des connaissances difficilement transmissibles à distance.

Nos résultats suggèrent donc que la baisse de la part de la mobilité dans les relations inter-entreprises passe notamment par une meilleure maîtrise des technologies avancées de communication à distance, et spécifiquement de l'Internet. 
L'investissement nécessaire à cette appropriation des technologies ne semble toutefois pas être une priorité pour les entreprises impliquées principalement dans des relations de proximité, la faiblesse des coûts des déplacements rendant moins nécessaire la rationalisation de la mobilité.

Parmi les facteurs avancés dans la littérature pour justifier le choix du mode de communication entre deux entreprises, plusieurs n'ont pas ici pu être testés. En particulier, la notion de proximité non spatiale, qui n'a pu ici être mesurée que par l'appartenance au même groupe et l'existence d'un contrat entre les deux partenaires, mérite d'être précisée. De même, la notion de «phases » dans la relation de coopération, qui, parce qu'elle implique notamment des échanges de connaissances de nature différentes, mais aussi parce qu'elle va jouer sur la connaissance mutuelle et donc sur la confiance entre les partenaires, peut être déterminante dans le choix du mode de communication, pourrait être introduite dans un prochain questionnaire via l'ancienneté de la relation de coopération.

\section{Bibliographie}

AGUILERA A., 2008, "Business travel and mobile workers", Transportation Research Part A, vol.42, n 9 , pp. 1109-1116.

AGUILERA, A., DE CONINCK, F., HAUCHARD, P., 2007, "Le rôle des déplacements professionnels dans les entreprises multi-établissements. Le cas d'un fournisseur de l'automobile", Recherche, Transports, Sécurité (RTS), pp. 195-209.

AMABILE S., GADILLE M., 2003, "Les NTIC dans les PME : stratégies, capacités organisationnelles et avantages concurrentiels", Revue française de Gestion, ${ }^{\circ}{ }^{144}$, pp. 43-63.

AMIN, A., 2002, "Spatialities of Globalization", Environment and Planning A, $\mathrm{n}^{\circ} 34$, pp. 385-399.

ANCORI B., BURETH A. COHENDET P., 2007, "The economics of knowledge: the debate about codification and tacit knowledge", Industrial and Corporate Change, vol.9, $\mathrm{n}^{\circ} 2$, pp. 255-287.

ARABEYRE-PETIOT A., 1999, "Le rôle des liaisons interurbaines dans l'arbitrage métropole-système productiif localisé. Cas de la sous-traitance aéronautique dans le sud-ouest de la France », Les Cahiers Scientifiques du Transport, n³6, pp. 4367.

ARNFALK P., KOGG B., 2003, "Service transformation- managing a shift from business travel to virtual meetings", Journal of Cleaner Production, 11(8), pp.859872.

BALCONI, M. POZZALI, A. AND VIALE, R., 2007, "The "codification debate" revisited: a conceptual framework to analyze the role of tacit knowledge in economics", Industrial and Corporate Change, vol. 16, n ${ }^{\circ}$, pp.823-849. 
BATHELT, H. MALMBERG, A. AND MASKELL, P., 2004, "Clusters and knowledge: local buzz, global pipelines and the process of knowledge creation", Progress in Human Geography, vol. 28, n 1, pp.31-56.

BOSCHMA R., 2005, «Proximity and innovation : A critical assessment », Regional Studies, vol.39, n¹, pp.61-74.

BOUBA-OLGA O., GROSSETI, M., 2008, "Socio-économie de proximité", Revue d'Economie Régionale et Urbaine, ${ }^{\circ} 3$, p. 311-328.

CARIOU C., LETHIAIS V. , 2006, Proximity, technology and mode of diffusion as determinants of knowledge flows, 5èmes Journées de la Proximité, Bordeaux.

CHARLOT S., DURANTON G., 2006, "Cities and workplace communication. Some quatitative french evidence", Urban Studies, vol. 43, n8, pp.1365-1394.

CLAISSE, G., ROWE, F., 1993, "Domestic telephone habits and daily mobility", Transportation research Part A, vol. 27, n4, pp. 277-289.

FERRU M., 2010, "La géographie des collaborations science-industrie: poids et impact des modalités de mise en relation”, Economie Appliquée, vol. 63, n¹, pp.105-134.

FILIPPI M., TORRE A., 2000, “L'organisation en réseau: de l'instauration de règles locales aux actions collectives", in DUPUY C., BURMEISTER A. (éds), Les nouveaux enjeux de la proximité, La Documentation Française, Paris, pp. 62-102.

GALLAUD, D. AND TORRE, A., 2005, "Geographical proximity and the diffusion of knowledge: The case of SMEs in biotechnology", in FUCHS G., SHAPIRA P. AND KOCH A. (éds), Rethinking Regional Innovation, Dordrecht: Kluwer.

GALLIANO, D. ET ROUX, P., 2006, “Les inégalités spatiales dans l'usage des TIC : le cas des firmes industrielles françaises", Revue Economique, vol. 57, nº, pp. 14491475.

GALLIANO, D., LETHIAIS V., SOULIE N., 2007, "Faible densité des espaces et usages des TIC par les entreprises : besoin d'information ou de coordination", Revue d'Economie Industrielle, vol. 57, nº, pp. 1449-1475.

GALLIE E.P., 2003, “Une grille d'analyse de l'usage des TIC dans les différentes étapes de la coopération technologique”, Sciences de la Société, n55.

GALLIE, E.P., GUICHARD R., 2005, "Do collaboratories mean the end of face to face interactions? An evidence from the ISEE Project", Economics of Innovation and New Technology, vol. 14, nº , pp. 517-532.

GASPAR J., GLEASER E. L., 1998, "Information Technology and the Future of Cities”, Journal of Urban Economics, n43, pp. 136-156.

GUILLAIN R., 2000, Villes et échanges d'information, Thèse en Sciences Economiques, Université de Bourgogne. 
HANDY, C., 1995, “Trust and Virtual Organization”, Harvard Business Review, vol. $73, \mathrm{n}^{\circ} 3$, pp. 40-50.

HILDRUM J., 2007, "When is frequent face-to-face contact necessary in innovation? A comparative study of two distributed product development projects », Economics of Innovation and New Technology, vol.16, $\mathrm{n}^{\circ} 6, \mathrm{pp} .467-484$

JOHNSON B., LORENZ E., LUNDVALL B.A., 2004, "Why all this fuss about codified and tacit knowledge?", Industrial and Corporate Change, vol.11, n², pp. 245-262.

KALIKA M., BOUKEF CHARKI M., ISAAC H., 2007, "La théorie du millefeuille et l'usage des TIC dans l'entreprise", Revue française de Gestion, vol. 33, n ${ }^{\circ} 172$, pp. 117-129.

KAUFMANN A., LEHNER P., TÖDTLING F., 2003, "Effects of the Internet on the spatial structure of innovation networks", Information Economics and Policy, vol. $15, \mathrm{n}^{\circ} 3, \mathrm{pp} .402-424$.

KNOBEN J., OERLEMANS L.A.G., 2006, "Proximity and inter-organizational collaboration: A literature review", International Journal of Management Review, vol.8, $\mathrm{n}^{\circ} 2$, pp.71-89.

LEBON S., BALLET B., 2004, "Le partenariat inter-entreprises dans le grand commerce et les services aux entreprises", INSEE Première, $\mathrm{n}^{\circ} 992$.

LE GOFF-PRONOST M., LETHIAIS V., 2008, "Usages des TIC et proximité géographique : une analyse empirique", Revue d'Economie Régionale et Urbaine, $\mathrm{n}^{\circ} 1, \mathrm{pp} .69-86$.

LORENTZON, S., 2003, "Changes in the Flows and Means of Information Exchange: Business Uses of ICT in Sweden in the 1990s", Journal of Urban Technology, vol.10, $\mathrm{n}^{\circ} 1$, pp. 89-110.

LING R., HADDON L., 2001, Mobile telephony, mobility and the coordination of everyday life, Paper presented at the conference "Machines that become us", Rutgers University, 18 and 19 April, 19 p.

LING R., YTTRI B., 2002, "Hyper-coordination via mobile phones in Norway", in KATZ J. AND AAKHUS M. (eds), Perpetual contact: Mobile communication, private talk, public performance, Cambridge University Press, Cambridge.

LUNDVALL, B.A. AND JOHNSON, B., 1994, "The Learning Economy", Industry and Innovation, vol. $1, \mathrm{n}^{\circ} 2$, pp. 23-42.

MASSOT M.H., 1995, Transport et télécommunications, collection Transports et communications, Paradigme, 248 p.

MOATI P., 2002, "De la très petite à la grande entreprise, état des lieux", Cahiers Français, n $^{\circ} 309$, pp. 368. 
MORGAN K., 2004, "The exaggerated death of distance: learning, proximity and territorial innovation systems", Journal of Economic Geography, n 4, pp. 3-21

RAllet A., AGUILERA A., GUILlOT C., "Diffusion des TIC et mobilité. Permanence et renouvellement des problématiques de recherche", Flux, nº78, pp. 7-16.

RALLET A., BURMEISTER A., 2002. Recherche sur la complémentarité des télécommunications et des transports et ses effets sur la localisation des activités et la mobilité des personnes, rapport pour la DRAST, octobre.

RALLET A., TORRE A., 2004, "Proximité et localisation”, Economie Rurale, n 280, pp.25-41.

SESSI, 2005, "Les partenariats des groupes industriels. Très fréquents, en interne comme en externe", Le 4 Pages des statistiques industrielles, $\mathrm{n}^{\circ} 206$, juillet.

TORRE A, 2008, "On the Role Played by Temporary Geographical Proximity in Knowledge Transmission”, Regional Studies, vol. 42, n6, pp.869-889

VECCHI A., WICKHMAN J., 2008, "Local Firms and Global Reach: Business Air Travel and the Irish Software Cluster", European Planning Studies, vol.16, n5, pp.693-710. 
Tableau 1: Nature des connaissances dans les relations de coopération (en pourcentage des entreprises)

\begin{tabular}{|c|c|c|c|c|} 
& $\begin{array}{c}\text { Informations } \\
\text { liées à votre } \\
\text { activités (know- } \\
\text { what) }\end{array}$ & $\begin{array}{c}\text { Savoir } \\
\text { scientifique } \\
\text { fondamental } \\
\text { (know-why) }\end{array}$ & $\begin{array}{c}\text { Savoir-faire } \\
\text { pratique } \\
\text { (know-how) }\end{array}$ & $\begin{array}{c}\text { Compétence ou } \\
\text { expertise } \\
\text { particulière } \\
\text { (know-who) }\end{array}$ \\
\hline Beaucoup & $73 \%$ & $19,5 \%$ & $54,9 \%$ & $45,7 \%$ \\
\hline Un peu & $18 \%$ & $14,1 \%$ & $20,1 \%$ & $19,5 \%$ \\
\hline Pas du tout & $9 \%$ & $66,4 \%$ & $25 \%$ & $34,8 \%$ \\
\hline Total & $100 \%$ & $100 \%$ & $100 \%$ & $100 \%$
\end{tabular}

Source : enquête M@rsouin, 2006 
Tableau 2 : Usages des différentes modes de communication dans les relations de coopération (en pourcentage des entreprises)

\begin{tabular}{|c|c|c|c|} 
& $\begin{array}{c}\text { Technologies } \\
\text { avancées }\end{array}$ & $\begin{array}{c}\text { Technologies } \\
\text { traditionnelles }\end{array}$ & $\begin{array}{c}\text { Rencontres } \\
\text { physiques }\end{array}$ \\
\hline Outil principal & $49.4 \%$ & $67,8 \%$ & $45,1 \%$ \\
\hline Outil secondaire & $19 \%$ & $24,4 \%$ & $37,6 \%$ \\
\hline $\begin{array}{c}\text { Outil le moins } \\
\text { important }\end{array}$ & $22,4 \%$ & $4,3 \%$ & $10.9 \%$ \\
\hline $\begin{array}{c}\text { N'utilise pas cet } \\
\text { outil }\end{array}$ & $9,2 \%$ & $3,4 \%$ & $6.3 \%$ \\
\hline Total & $100 \%$ & $100 \%$ & $100 \%$
\end{tabular}

Source : enquête M@rsouin, 2006 
Tableau 3: Fréquence d'utilisation des différents moyens de communication (en pourcentage des entreprises)

\begin{tabular}{|c|c|c|c|c|c|c|c|} 
& $\begin{array}{c}\text { Rencontre } \\
\text { s } \\
\text { physiques }\end{array}$ & Téléphone & $\begin{array}{c}\text { Courrier } \\
\text { fax }\end{array}$ & Mail & $\begin{array}{c}\text { Visio- } \\
\text { conf }\end{array}$ & $\begin{array}{c}\text { Outils } \\
\text { collab }\end{array}$ & $\begin{array}{c}\text { Outils de } \\
\text { gestion } \\
\text { des collab }\end{array}$ \\
\hline Quoti & $9,2 \%$ & $43,7 \%$ & $40,5 \%$ & $50,9 \%$ & $0,9 \%$ & $22,4 \%$ & $8,6 \%$ \\
\hline Hebdo & $15,8 \%$ & $34,5 \%$ & $28,7 \%$ & $22,7 \%$ & $1,2 \%$ & $12,7 \%$ & $5,2 \%$ \\
\hline Mensuelle & $33 \%$ & $12,9 \%$ & $11,2 \%$ & $10 \%$ & $3,2 \%$ & $8,9 \%$ & $4,3 \%$ \\
\hline $\begin{array}{c}\text { Moins } \\
\text { souvent }\end{array}$ & $33 \%$ & $3,7 \%$ & $10,3 \%$ & $5,2 \%$ & $7,5 \%$ & $11,5 \%$ & $11,8 \%$ \\
\hline Jamais & $8,9 \%$ & $5,2 \%$ & $9,2 \%$ & $11,2 \%$ & $87,4 \%$ & $45,1 \%$ & $70,1 \%$ \\
\hline Total & $100 \%$ & $100 \%$ & $100 \%$ & $100 \%$ & $100 \%$ & $100 \%$ & $100 \%$
\end{tabular}

Source : enquête M@rsouin, 2006 


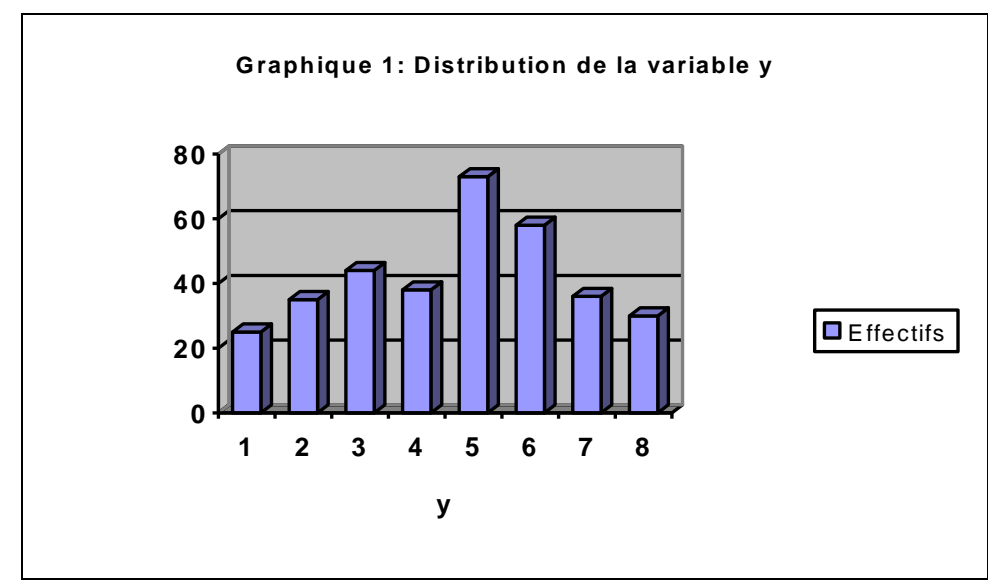


Tableau 4 : Résultats modèles économétriques : estimation de la fréquence relative des rencontres physiques dans les relations de coopération

\begin{tabular}{|c|c|c|c|}
\hline & $\begin{array}{c}\text { Modèle } 1: \\
\text { Echantillon total }\end{array}$ & $\begin{array}{c}\text { Modèle } 2 \text { : } \\
\text { Relations régionales }\end{array}$ & $\begin{array}{c}\text { Modèle } 3 \text { : } \\
\text { Relations hors région }\end{array}$ \\
\hline \multicolumn{4}{|l|}{ Importance des autres outils } \\
\hline Technologies traditionnelles & -0.1744 & 0.1122 & -0.4281 \\
\hline Nouvelles technologies & $-0.5183 * *$ & -0.4195 & $-0.6986 * *$ \\
\hline \multicolumn{4}{|l|}{ Vecteurs d'échanges } \\
\hline Documents et fichiers & $-0.5543 * *$ & $-0.7143 * *$ & -0.3008 \\
\hline Echanges oraux & $0.7606 * * *$ & $0.8190 * *$ & $0.7268 * * *$ \\
\hline \multicolumn{4}{|l|}{ Types de connaissances } \\
\hline Informations & $-1.4530 * * *$ & $-2.0171 * * *$ & $-1.4368 * *$ \\
\hline Savoir scientifique & $-2.2185 * *$ & -2.1792 & $-3.4750 * *$ \\
\hline Savoir-faire pratique & $-1.4084 * *$ & -1.4479 & $-1.8264 * *$ \\
\hline Compétences ou expertises & Réf. & Réf. & Réf. \\
\hline \multicolumn{4}{|l|}{ Localisation du partenaire } \\
\hline Même zone d'activité & $1.3366 * * *$ & $0.9890 * *$ & \\
\hline Echelon local & $1.1158 * * *$ & 0.5864 & \\
\hline Echelon régional & 0.1759 & Réf. & \\
\hline Echelon national & 0.1990 & - & 0.2556 \\
\hline Echelon international & Réf. & - & Réf. \\
\hline \multicolumn{4}{|l|}{ Proximités du partenaire } \\
\hline Appartenance au groupe & 0.2448 & 0.1484 & 0.3356 \\
\hline Contractualisation & -0.1401 & -0.4868 & 0.0591 \\
\hline $\begin{array}{l}\text { Nombres de relations }(+\mathrm{de} \\
10)\end{array}$ & $-0.3785 *$ & -0.0120 & $-0.6638 * *$ \\
\hline \multicolumn{4}{|c|}{ Appropriation des technologies } \\
\hline Diffusion de l'informatique & $-0.6865 * *$ & $-1.1824 * *$ & -0.2265 \\
\hline Apprentissage d'Internet & $-0.4876 * *$ & $-0.6114 *$ & -0.3748 \\
\hline Nombre d'observations & 339 & 131 & 208 \\
\hline $\begin{array}{l}\text { Pourcentage de } \\
\text { concordance }\end{array}$ & 68.2 & 72.2 & 64.7 \\
\hline
\end{tabular}

Source : enquête M@rsouin, 2006 